\title{
FLOW CYTOMETRIC IMMUNOPHENOTYPING OF ACUTE LEUKAEMIA WITH SPECIAL REFERENCE TO MIXED PHENOTYPIC ACUTE LEUKAEMIA
}

\author{
Nitu Mani Khakhlari', Bibhash Gogoi², Abhinanda Barua3, Vishal Agarwal4 \\ ${ }^{1}$ Associate Professor, Department of Pathology, Jorhat Medical College and Hospital, Jorhat, Assam. \\ ${ }^{2}$ Assistant Professor, Department of Pathology, Jorhat Medical College and Hospital, Jorhat, Assam. \\ ${ }^{3}$ Demonstrator, Department of Pathology, Gauhati Medical College and Hospital, Guwahati, Assam. \\ ${ }^{4}$ Demonstrator, Department of Pathology, Assam Medical College and Hospital, Barbari, Dibrugarh, Assam.
}

ABSTRACT

\section{BACKGROUND}

Acute leukaemias are classified according to their characterisation of either the myeloid or lymphoid lineage. Immunophenotyping for various intracellular and extracellular cell lineage specific markers is an important feature in differentiating between acute myeloid leukaemia (AML) and acute lymphoid leukaemia (ALL). In about 5\% cases of Acute Leukaemia, it is not clear whether blasts are derived from myeloid or lymphoid progenitors and are classified as Mixed Phenotypic Acute Leukaemia (MPAL). Our study aimed to analyse the prevalence of MPAL.

\section{MATERIALS AND METHODS}

We prospectively investigated the phenotype of blast cells from 183 cases of acute leukaemia patients using a large panel of monoclonal antibodies by multiparametric flow cytometry.

\section{RESULTS}

183 cases of acute leukaemia were analysed using multiparametric flow cytometry. Out of which 115 cases (62.84\%) were Acute Myeloid Leukaemia (AML), 49 cases (26.77\%) were B cell Acute Lymphocytic Leukaemia (B-ALL), 8 cases (4.37\%) were T cell Acute Lymphocytic Leukaemia (T-ALL) and 11 cases (6.01\%) were Mixed Phenotypic Acute Leukaemia (MPAL). Out of 11 cases of MPAL, 5 cases (45.45\%) were in paediatric age group and 6 cases (54.55\%) were in adult age group. 8 cases (72.73\%) of MPAL were male patients and 3 cases (27.27\%) of MPAL were female patients. Of the MPAL cases, 10 cases of B+Myeloid type and 1 case of $\mathrm{T}+$ Myeloid were found. No B+T+Myeloid or B+T type MPAL was found.

\section{DISCUSSION}

Our study demonstrates that Mixed Phenotypic Acute leukaemia is a rare form of Acute Leukaemia. It represents a subgroup with a worse prognosis. These patients present more often with extramedullary localisation of disease, which urges for more intensive treatment protocols including central nervous system prophylaxis.

\section{CONCLUSION}

Our study concludes that Mixed Phenotypic Acute Leukaemia is a rare type of acute leukaemia with heterogeneous clinical, immunophenotypic and genetic characteristics and has got a poor prognosis than the other acute leukaemias.

\section{KEYWORDS}

Mixed Phenotypic Acute Leukaemia, Acute Leukaemia, Flow cytometry.

HOW TO CITE THIS ARTICLE: Khakhlari NM, Gogoi B, Barua A, et al. Flow cytometric immunophenotyping of acute leukaemia with special reference to mixed phenotypic acute leukaemia. J. Evolution Med. Dent. Sci. 2017;6(32):2585-2589, D0I: $10.14260 /$ Jemds/2017/559

\section{BACKGROUND}

The diagnosis and classification of acute leukaemia is based on a multidisciplinary approach which includes the morphology, cytochemistry, immunophenotyping, karyotyping and molecular genetic analysis. By using this multidisciplinary approach, most acute leukaemias can be classified into myeloid, B-lymphoid or T-lymphoid lineage.

Financial or Other, Competing Interest: None.

Submission 14-03-2017, Peer Review 07-04-2017,

Acceptance 14-04-2017, Published 20-04-2017.

Corresponding Author:

Dr. Nitu Mani Khakhlari,

Associate Professor,

Department of Pathology,

Jorhat Medical College and Hospital,

Jorhat, Assam.

E-mail: nitumanikhakhlari@gmail.com

DOI: $10.14260 /$ jemds $/ 2017 / 559$

\section{(c) $($ ) $\$$}

Multiparametric flow cytometry is the preferred method of immunophenotypic analysis in Acute Leukaemia due to the ability to analyse large numbers of cells in a relatively short period of time with simultaneous recording of information about several antigens for each individual cell. Evaluation of expression patterns of several antigens, both membrane and cytoplasmic, is necessary for lineage assignment, to detect mixed phenotype acute leukaemia and to detect aberrant phenotypes allowing for follow-up of minimal residual disease. ${ }^{1}$

However, even after extensive immunophenotyping using a broad panel of antibodies, a small and heterogeneous subset of leukaemias cannot be assigned to a particular group i.e. myeloid, $\mathrm{B}$, or $\mathrm{T}$ lymphoid. Leukaemias under this category were given many different names, including bilineage leukaemias, biphenotypic leukaemias, hybrid leukaemias, undifferentiated leukaemias. The term acute bilineage leukaemia has been applied to leukaemias 
containing separate populations of blasts of more than one lineage and the term biphenotypic leukaemia to those containing a single population of blasts co-expressing antigens of more than one lineage. ${ }^{2}$

Later, the European Group for the Immunological Characterisation of Leukaemias (EGIL) proposed an immunological classification and characterisation of acute leukaemias, including a definition for biphenotypic acute leukaemia in 1995 and was later integrated in the 2001 WHO classification. ${ }^{3}$

In 2008 WHO classification, bilinear and biphenotypic acute leukaemias were grouped together under a new heading, Mixed Phenotypic Acute Leukaemia (MPAL). WHO definition of MPAL is based on the expression of strictly specific T-lymphoid (cytoplasmic CD3) and myeloid (myeloperoxidase [MPO]) antigens, the latter shown by flow cytometry (FCM) or cytochemistry and/or clear evidence of monocytic differentiation. Because there is no single antigen strictly specific for B cells, B-cell lineage assignment in MPAL relies on the strong expression of CD19 together with another B cell-associated marker or, in cases, with weak CD19, on the expression of at least $3 \mathrm{~B}$-lineage markers. ${ }^{4}$

\section{MATERIALS AND METHODS}

The study was carried out in the DBT Health Care Flow cytometry Laboratory, Department of Pathology, Assam Medical College, Assam, India for a period of four years from June, 2012 to May, 2016. A total number of 183 cases of acute leukaemia were included in the study, which were subjected to routine haematological investigations and cytochemistry followed by multiparametric flow cytometry. Diagnosis of acute leukaemia was made on routinely stained bone marrow aspiration and blood smears. Immunophenotyping was carried out on bone marrow or peripheral blood.

Complete blood count was done by using Sysmex XS-800i and peripheral blood film stained by Giemsa stain to find the presence of blast cells as shown in Figure 1. A total of 500 cells of WBC were counted and blasts cells over $20 \%$ are regarded as acute leukaemias. Then whole blood or aspirate samples were prepared by cell Stain-Lyse-Wash method for immunofluorescence staining with different antibodies which were conjugated with fluorochromes (i.e. APC H7, PE cy7, FITC, PE, APC and PerCPcy5.5). Cell washing was done with phosphate buffer saline (PBS) (NaH2PO4.2H2O, Na2HPO4 and $\mathrm{NaCl}$ ). When whole blood is added to the monoclonal antibody reagent, the fluorochrome labelled antibodies in the reagent bind specifically to leucocyte surface antigens. The stained samples were then treated with FACS lysing solution (NH4CL) which lyses erythrocytes under gentle hypotonic conditions while preserving the leucocytes.

The permeabilizing solution containing $15 \%$ formaldehyde and 50\% diethylene glycol and proprietary permeabilizing agent used for intracellular staining of antigens such as MPO, CD79a, CD3 Cytoplasmic and Tdt. Data acquisition and analysis were performed on a FACS Canto 2 flow cytometer (Becton Dickinson, San José, USA) using BD FACS Diva software. Identification of blast cells was performed using side scatter (SSC) versus CD45 intensity and SSC versus forward scatter (FSC) parameter dot plots. The percentage of gated abnormal population expressing a particular CD marker was analysed whether expression was positive or negative ( $>20 \%$ for surface antigen and $>10 \%$ for cytoplasmic antigen). The positivity and the negativity was analysed considering both the quadrant gate as well as the intensity of expression.

Acute leukaemia cases were classified into Acute Myeloid Leukaemia (AML), B cell Acute Lymphocytic Leukaemia (B-ALL), T cell Acute Lymphocytic Leukaemia (T-ALL) and Mixed Phenotypic Acute Leukaemia (MPAL). MPAL cases were diagnosed by 2008 WHO criteria as shown in Table 1.4

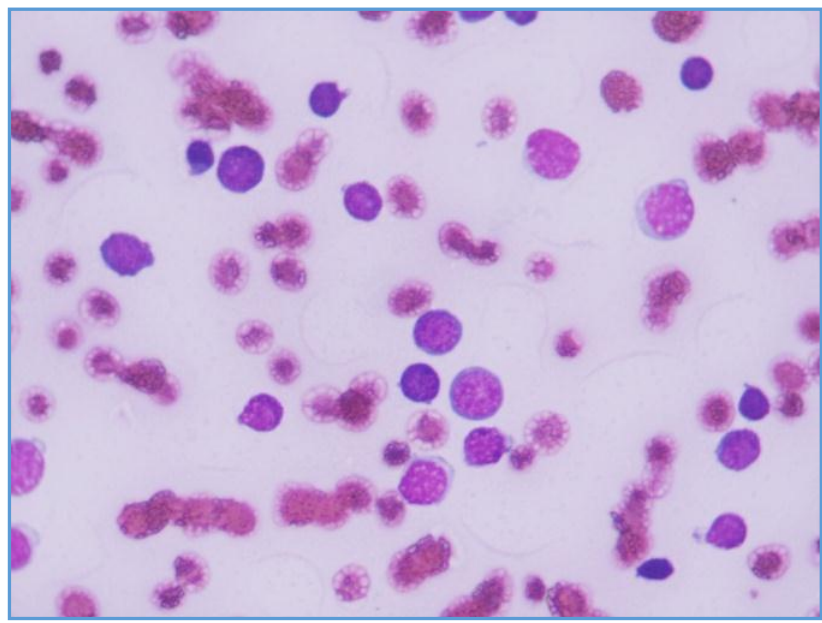

Figure 1. Giemsa stained Peripheral Blood Smear showing a Mixed-cell Population of large and Small Blasts

\begin{tabular}{|c|c|}
\hline Lineage & Markers \\
\hline Myeloid & $\begin{array}{c}\text { Myeloperoxidase or Monocytic differentiation } \\
\text { (At least two of the following: NSE, CD11c, CD14, } \\
\text { CD64, lysozyme) }\end{array}$ \\
\hline T Lineage & Cytoplasmic CD3 or Surface CD3 \\
\hline B Lineage & $\begin{array}{r}\text { Strong CD19 and at least one of the following with } \\
\text { strong expression: CD79a, } \\
\text { cytoplasmic CD22 or CD10 } \\
\text { OR } \\
\end{array}$ \\
$\begin{array}{c}\text { Weak CD19 and at least two of the following with } \\
\text { strong expression: CD79a, cytoplasmic CD22 or } \\
\text { CD10 }\end{array}$ \\
\hline \multicolumn{2}{|c|}{ Table 1. 2008 $\mathbf{W H O}$ Diagnostic Criteria for $\mathbf{M P A L}^{\mathbf{4}}$} \\
\hline
\end{tabular}



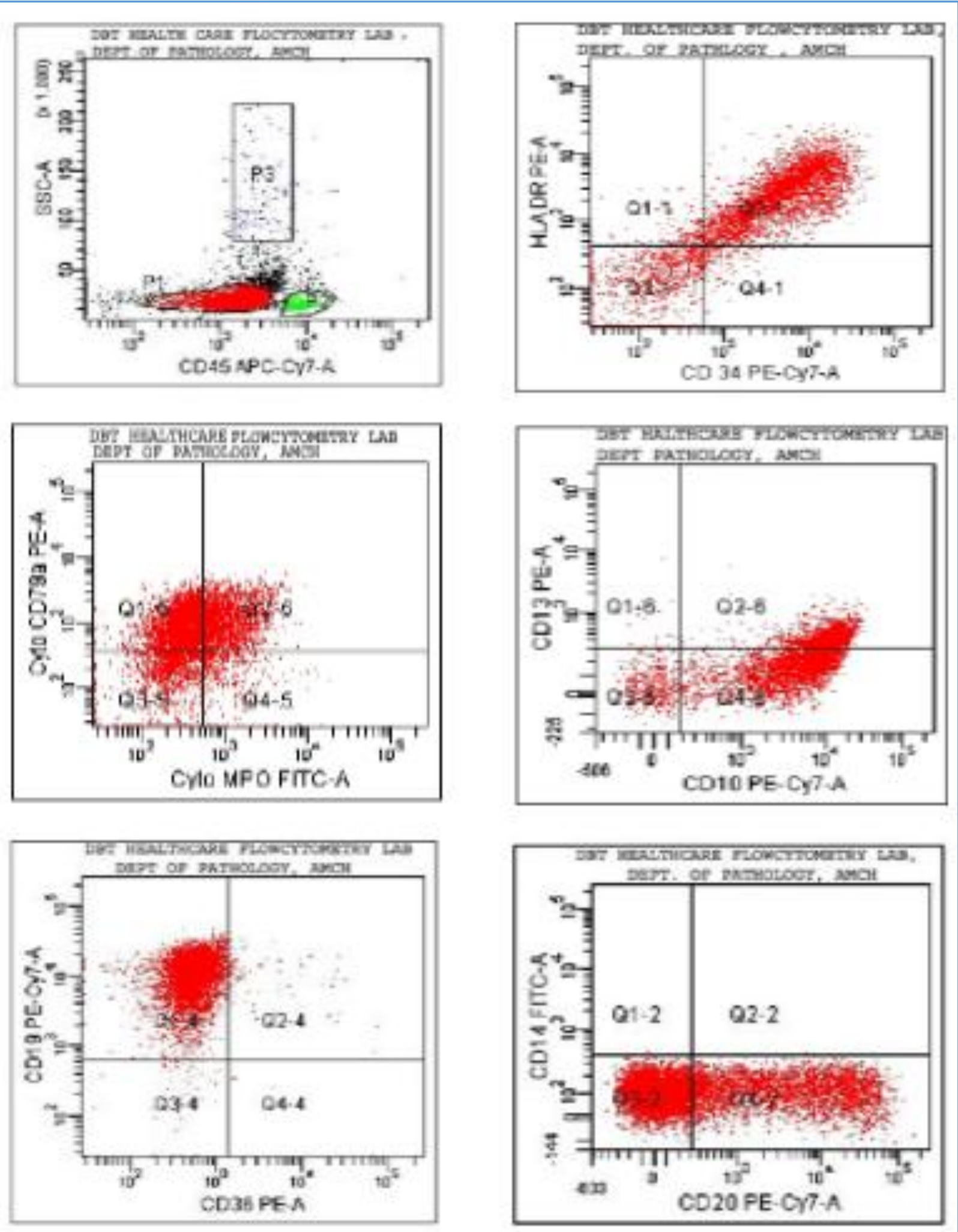

Figure 2. Dot plot Showing the Expression of both Myeloid and Lymphoid Markers

\section{RESULTS}

Acute leukaemia has been classified into AML, B-ALL, T-ALL and MPAL by flow cytometric immunophenotyping. In the present study, 183 cases of acute leukaemia were found. Out of which, 115 cases (62.84\%) of AML, 49 cases (26.77\%) of BALL, 8 cases $(4.37 \%)$ of T-ALL and 11 cases $(6.01 \%)$ of MPAL were found as shown in Table 2.

\begin{tabular}{|c|c|}
\hline Types & No. of Cases (\%) \\
\hline AML & $115(62.84)$ \\
\hline B-ALL & $49(26.77)$ \\
\hline T-ALL & $8(4.37)$ \\
\hline MPAL & $11(6.01)$ \\
\hline Table 2. Flow cytometric Immunophenotyping \\
of Acute Leukaemia
\end{tabular}


Out of 11 cases of MPAL, 5 cases (45.45\%) were in paediatric age group and 6 cases (54.55\%) were in adult age group as shown in Figure 3. We got 8 cases (72.73\%) of MPAL in male patients and 3 cases $(27.27 \%)$ of MPAL in female patients, Figure 4.

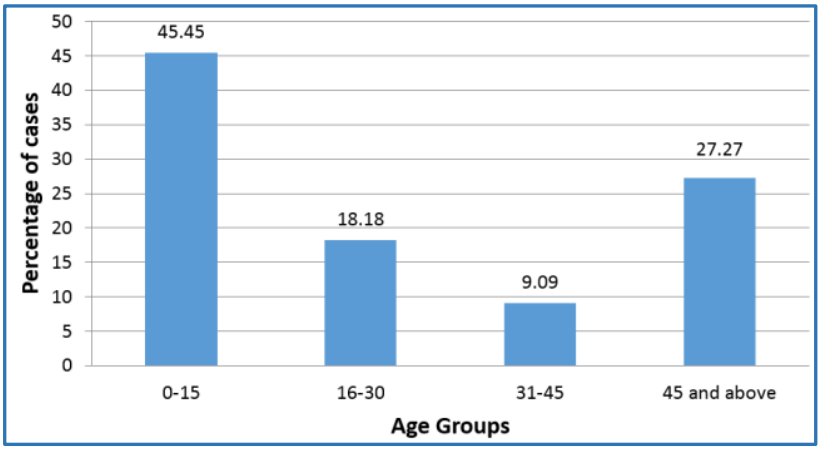

Figure 3. Age Distribution of MPAL Cases

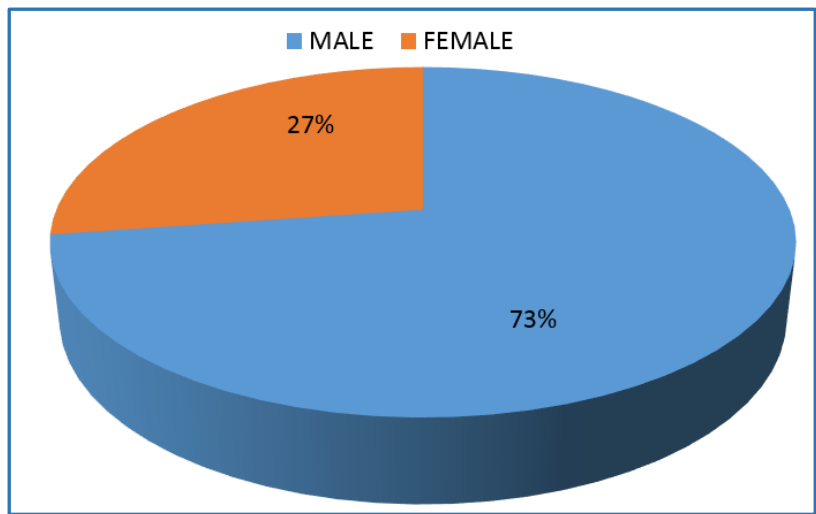

Figure 4. Sex Distribution of MPAL Cases

Of the MPAL cases, 10 cases of B+Myeloid type and 1 case of $\mathrm{T}+$ Myeloid type MPAL were found. No B+T+Myeloid and $\mathrm{B}+\mathrm{T}$ type MPAL was found as shown in Table 3.

\begin{tabular}{|c|c|}
\hline Types & No. of Cases (\%) \\
\hline B+Myeloid & $10(90.91)$ \\
\hline T+Myeloid & $1(9.09)$ \\
\hline B+T+Myeloid/ B+T & 0 \\
\hline \multicolumn{2}{|c|}{ Table 3. Different types of MPAL } \\
\hline
\end{tabular}

\section{DISCUSSION}

Most of acute leukaemias can be classified as myeloid, B lymphoid and $\mathrm{T}$ lymphoid based on different antigenic expression of the blast cells. However, some of the blast cells express both lymphoid and myeloid lineage-specific antigens, so lineage assignment in this minority of cases is not possible. These cases have been mentioned in the literature as biphenotypic acute leukaemia or mixed-lineage acute leukaemias and, recently, the designation of MPAL has been proposed by the WHO.4

Earlier, a diagnosis of biphenotypic acute leukaemia was made using the few available lineage associated markers, which had spuriously shown an incidence of acute mixed leukaemia to be as high as $20 \% .^{5}$ As more antibodies became available, it became clear that a significant proportion of AML and ALL cases expressed aberrant antigens. ${ }^{6,7}$ It became necessary to distinguish aberrant expression of an antigen(s) from true "mixed phenotype acute leukaemia". Specific criteria were first enunciated by Catovsky et $\mathrm{al}^{8}$ in the form of a scoring system based on weighted expression of antigens by blasts. This scoring system was further modified by EGIL where a score of over 2 was required to assign a lineage. The WHO 2008 classification, however, has dismissed these scoring systems and relies upon specific lineage assignment. Using this recent WHO criterion, prevalence of MPAL has been found to be less than $4 \%$ of acute leukaemia. 4

MPAL includes rather heterogeneous acute leukaemia cases. It affects both adults and children, including infants, although it seems more frequent in adults. There is a slight male predominance. There was no correlation between immunophenotypic subtypes and sex or age. Morphologically, MPAL is heterogeneous and most patients present either as ALL or as AML with or without myeloid or monocytic differentiation features. Thus, a diagnosis of MPAL is unlikely to be suspected by morphology except for the small subset in which there is evidence of a distinct dual-blast population with either lymphoid or myeloid features (Figure 1). Therefore, the diagnosis of MPAL always relies on immunophenotyping and exclusion by cytogenetics of AML cases with recurrent abnormalities and by morphology that rules out the presence of a dysplastic background.

In our study, we have found $6.01 \%$ cases of MPAL; however, Ancker et $\mathrm{al}^{9}$ found the prevalence of MPAL to be $5.0 \%$. Out of 11 cases of MPAL, 5 cases (45.45\%) were in paediatric age group ( $<15$ years) and 6 cases $(54.55 \%)$ were in adult age group is similar to Owaidah TM et al. ${ }^{10}$ They found 11 patients $(47.82 \%)$ were aged 14 years or less and the other 12 cases $(52.17 \%)$ were adults.

In our study, we found 8 cases $(72.73 \%)$ of MPAL in males and 3 cases $(27.27 \%)$ of MPAL in females out of 11 cases whereas Estella et al ${ }^{11}$ noted $62(62.0 \%)$ males and 38 (38.0\%) females out of 100 cases of MPAL.

In the present study, 10 cases $(90.90 \%)$ of B+Myeloid type and 1 case $(9.09 \%)$ of $\mathrm{T}+$ Myeloid type MPAL were found. No B+T+Myeloid and B+T type MPAL was found. All the large series of studies by Estella et al ${ }^{11}$ found B+Myeloid (59\%), $\mathrm{T}+$ Myeloid (35\%), B+T (4\%), or $\mathrm{B}+\mathrm{T}+$ Myeloid $(2 \%)$; and Lingzhi et al ${ }^{12}$ found $\mathrm{B}+$ Myeloid $(54.7 \%), \mathrm{T}+$ Myeloid (32.47\%), B+T (11.96\%) and B+T+Myeloid (0.85\%).

\section{CONCLUSION}

To conclude, MPAL is a rare type of acute leukaemia accounting for less than $5 \%$ of cases. It is a complex entity with heterogeneous clinical, immunophenotypic and genetic characteristics. It has poor prognosis than AML and ALL cases. It is necessary to conduct multicentric studies to determine optimal regimens for induction and consolidation therapy and to define the role of HSCT in MPAL.

\section{ACKNOWLEDGEMENTS}

We acknowledge Department of Biotechnology, Govt. of India. We further acknowledge Miss Rashmi Roy, JRF; Mr Sautom Roy, Data entry operator; Mr Pranjal Saikia and Mr Gautam Saikia, Technician; Mrs Labonya Gogoi, Lab attendant.

\section{REFERENCES}

[1] Weinberg OK, Arber DA. Mixed-phenotype acute leukemia-historical overview and a new definition. Leukemia 2010;24(11):1844-51. 
[2] Weir EG, Borowitz MJ. Acute leukemias of ambiguous lineage. In: Jaffe ES, Harris NL, Vardiman JW, et al. eds. Hematopathology. Philadelphia: Elsevier 2010.

[3] Bene MC, Castoldi G, Knapp W, et al. Proposals for the immunological classification of acute leukemias. European Group for the Immunological Characterization of Leukemias (EGIL). Leukemia 1995;9(10):1783-6.

[4] Borowitz M, Bene MC, Harris NL, et al. Acute leukemias of ambiguous lineage. In: Swerdlow $\mathrm{SH}$, Campo E, Harris NL, et al. eds. World Health Organization classification of tumours: pathology and genetics of tumours of haematopoietic and lymphoid tissues. Lyon, France: IARC Press 2008:150-5.

[5] Mirro J, Zipf TF, Pui $\mathrm{CH}$, et al. Acute mixed lineage leukemia: clinicopathologic correlation and prognostic significance. Blood 1985;66(5):1115-23.

[6] Porta DMG, Lanza F, Vecchio DL, et al. Flow cytometry immunophenotyping for the evaluation of bone marrow dysplasia. Cytometry B Clin Cytom 2011;80(4):201-11.

[7] Haycocks NG, Lawrence L, Cain JW, et al. Optimizing antibody panels for efficient and cost-effective flow cytometric diagnosis of acute leukemia. Cytometry B Clin Cytom 2011;80B(4):221-9.
[8] Catovsky D, Matutes E, Buchheri V, et al. A classification of acute leukemia for the 1990s. Ann Hematol 1991;62(1):16-21.

[9] van den Ancker W, Terwijn M, Westers TM, et al. Acute leukemias of ambiguous lineage: diagnostic consequences of the WHO 2008 classification. Leukemia 2010;24(7):1392-6.

[10] Owaidah TM, Al Beihany A, Iqbal MA, et al. Cytogenetics, molecular and ultrastructural characteristics of biphenotypic acute leukemia identified by the EGIL scoring system. Leukemia 2006;20(4):620-6.

[11] Matutes E, Pickl WF, van't Veer M, et al. Mixedphenotype acute leukemia: clinical and laboratory features and outcome in 100 patients defined according to the WHO 2008 classification. Blood 2011;117(11):3163-71.

[12] Yan L, Ping N, Zhu $\mathrm{M}$, et al. Clinical, immunophenotypic, cytogenetic and molecular genetic features in 117 adult patients with mixed-phenotype acute leukemia defined by WHO-2008 classification. Haematologica 2012;97(11):1708-12. 